\title{
EDITORIAL \\ Sodium values and the use of hyperosmolar therapy following traumatic brain injury
}

\author{
Gregory W. J. Hawryluk, MD, PhD, FRCSC \\ Department of Neurological Surgery, University of Utah, Salt Lake City, Utah
}

$\mathrm{W}$ HEREAS other areas of medicine have entered an apparent golden era of personalized medicine, the neurotrauma field is still struggling with comparatively basic issues. The landmark work of Weed and McKibben (see Wilkins et al.) established hyperosmolar therapy as an effective treatment for intracranial hypertension a century ago. ${ }^{20}$ This work was disruptive because it introduced the new notion that the brain could change its volume. A century before that-long before Cajal's work and the advent of neuron theory-the writings of Monro ${ }^{16}$ and Kellie ${ }^{11}$ espoused the belief that the volume of the brain was constant.

Despite the unquestionable and marked efficacy of this life-saving therapeutic strategy, insufficient literature has come forth over the last 100 years to inform the best use of hyperosmolar therapy. Indeed, the recently published fourth edition of the Brain Trauma Foundation (BTF) Guidelines for the Management of Severe Traumatic Brain Injury (TBI) failed to find any evidence meeting current standards on which to base recommendations. ${ }^{8}$ Thus, there is a tremendous need to better understand the different properties of hypertonic saline and mannitol and how these agents are best administered to the critically ill patients who require them.

Although I acknowledge the substantial deficiencies in the literature as well as opinions that differ from my own, I have been concerned with some practices related to hyperosmolar therapy that seem increasingly prevalent and that are discordant with important laboratory and clinical evidence that we do have-as well as the subspecialty training I received from leading figures in the field of neurotrauma. First, it is my impression that liberal and even prophylactic administration of hyperosmolar therapy is increasingly commonplace. In my region, first responders now frequently administer hypertonic saline to normotensive patients suspected of having intracranial pathology but who do not show signs of herniation or a progressive neurological deficit. This is despite the recommendations in the BTF's guidelines not to do so because of the harm associated with the prophylactic treatment of intracranial pressure with hyperosmolar and other therapies. ${ }^{4,8}$

In this regard, it is important for clinicians to be aware of laboratory investigations that have demonstrated that homeostatic mechanisms see the increased production of osmoles by the brain in hypernatremic or hyperosmolar states. ${ }^{10,14}$ These additional brain osmoles aim to maintain the volume of the brain and reduce the concentration gradient between the brain and the administered hyperosmolar therapies. This phenomenon is referred to as brain adaptation, and it has been extensively studied and described in the older literature. ${ }^{10}$ The volume of water that will cross a semipermeable membrane is, of course, dependent on the concentration gradient between the two compartments. On the basis of theory as well as experimental observations, there is thus concern that prophylactic administration of hyperosmolar therapy makes it less effective when its administration is truly required.

A highly related second concern that I have is the practice of sodium targeting-or trying to achieve and maintain a specific serum sodium concentration in the high normal or supranormal range in hopes of avoiding or reducing intracranial hypertension. The concern with sodium targeting is that it is at least to a certain degree prophylactic, invoking the issues described above. An additional concern is with continuous (non-bolus) hyperosmolar therapy infusions that are often prescribed as part of sodium targeting strategies. Here the issue is that the small concentration gradient generated limits the efficacy of this approach. Indeed, continuous infusion of hyperosmolar therapy for the management of intracranial pressure is a contravention of the American College of Surgeons' Trauma Quality Improvement Program consensus statements 
on $\mathrm{TBI},{ }^{3}$ which instead recommend intermittent boluses. I also note that the second edition of the BTF's adult management guidelines recommended against the continuous infusion of mannitol, 7 although the evidence was of insufficient strength to be carried forward in subsequent BTF guidelines. Of note, the concern with continuous mannitol infusion is not only its limited efficacy but also increased deposition of mannitol in brain parenchyma, which occurs with continuous as compared with intermittent dosing and can exacerbate and prolong brain edema. Without question, we need more high-quality research to inform these issues, but I belong to a group of neurotraumatologists who believe that these prevalent practices are in discordance with the best evidence currently available.

Of great relevance to the issue of liberal hyperosmolar administration and resulting hypernatremia is an article in this edition of Neurosurgical Focus that may cause us to consider more carefully serum sodium values and hyperosmolar therapy management following severe TBI. Indeed, this work was recently awarded Best Poster at the 2017 National Neurotrauma Symposium by the AANS/ CNS Joint Section on Neurotrauma and Critical Care. I have great respect for Claudia Robertson and the impactful contributions that she and her colleagues have made to our discipline. A common theme emanating from this group's important works has been the repeated demonstration of practitioners taking something assumed to be good too far-in violation of Milton's "rule of Not too much." To date, her group has demonstrated that routinely pushing the cerebral perfusion pressure above $70 \mathrm{~mm} \mathrm{Hg}$ is inadvisable, and that transfusion of blood to a hemoglobin concentration of $10 \mathrm{~g} / \mathrm{dl}$ or greater is similarly inadvisable after TBI. This new paper by Vedantam et al. ${ }^{19}$ promises to do the same for serum sodium values.

In their paper, Vedantam et al..$^{19}$ associated high serum sodium with poor outcome. This is not new. ${ }^{2,13,15}$ What is new and important about this paper, however, is the fact that the authors demonstrated this association after extensively addressing confounding factors that impeded the interpretation of preceding studies. In these prior studies, the hypernatremia of many of the patients studied was attributable to diabetes insipidus resulting from a more severe injury. In the present retrospective analysis, patients with diabetes insipidus were excluded, and key confounding variables such as injury severity were controlled for in the group's statistical models. The authors ultimately demonstrated a robust dose-dependent relationship between higher sodium values and outcome. Remarkably, this was demonstrated in both patients who received and patients who did not receive hyperosmolar therapies. Moreover, the number of hyperosmolar therapy doses failed to demonstrate a relationship with outcome, suggesting that this therapy was not the harmful entity. Perhaps most concerning was the magnitude of the association of hypernatremia with poor outcome - a statistically significant hazard ratio for mortality of 8.4 for patients in the severe hypernatremia group.

This paper has important limitations. It is retrospective, and residual confounding is likely to be problematic at least to some degree. Moreover, mannitol was exclusively used as the hyperosmolar agent. Nonetheless, I think that this important paper mandates discussion and additional

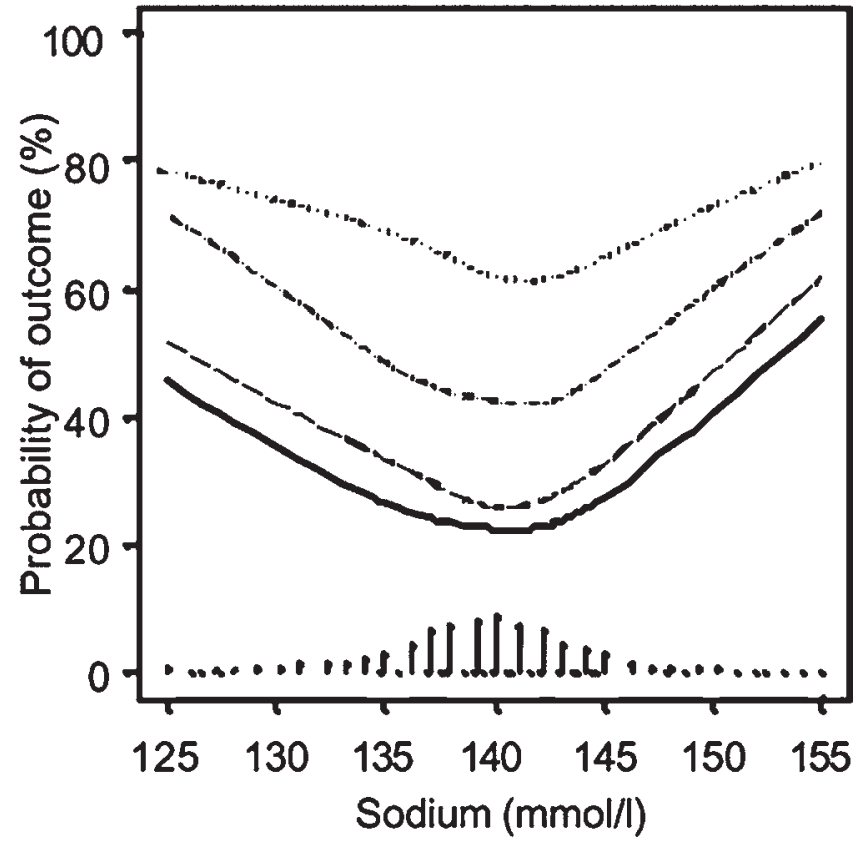

FIG. 1. The relationship between serum sodium values and outcome at 6 months demonstrated in the IMPACT study (Van Beek et al.). Spline functions are plotted-the lowest line (solid) indicates the probability of mortality (Glasgow Outcome Scale [GOS] Score 1); the second the combination of mortality and vegetative state (GOS 1 or 2); the third an unfavorable outcome (GOS 1, 2, or 3); and the fourth line the probability of a less than good outcome (GOS < 5). The publisher for this copyrighted material is Mary Ann Liebert, Inc., publishers. Figure reproduced with permission.

research, and should cause us to question what we think we know about sodium values after TBI. Moreover, I think it must cause us to question the extent to which hypernatremia has a harmful effect and plays a causative role in poor outcomes.

I think that without question the best look that we have had at the univariate association between serum sodium and outcome following TBI was published in conjunction with the landmark International Mission for Prognosis and Clinical Trial (IMPACT) study ${ }^{18}$ (Fig. 1), with which the data presented by Vedantam et al. are consistent. The analysis of "big data" in the IMPACT study allowed the continuous association between serum sodium and outcome. My interpretation of the spline functions presented in that study is that a sodium level of approximately $142 \mathrm{mmol} / \mathrm{L}$ may be associated with the best outcome from TBI.

In my opinion, the study by Vedantam et al..$^{19}$ should particularly cause us to scrutinize the sodium limits associated with hyperosmolar therapies. A serum sodium level of $155 \mathrm{mEq} / \mathrm{L}$ (and osmolality of $320 \mathrm{mOsm} / \mathrm{L}$ ) is generally considered to be the upper limit at which mannitol can be safely administered. Some practitioners use a higher limit for hypertonic saline-a serum sodium level of 160 $\mathrm{mmol} / \mathrm{L}$ and osmolality as high as $360 \mathrm{mOsm} / \mathrm{L}$, although some prefer the lower safety limits for both agents. ${ }^{9}$ Of course, the appropriate thresholds would be those at which serum sodium and osmolality are associated with greater harm than the intracranial pressure they aim to treat. In this context, there is a strong possibility that the ideal thresholds are specific to the patient and the situation. 
Finding published data supporting these established thresholds for safe hyperosmolar administration requires extensive digging in the old literature. For mannitol, the osmolality threshold of $320 \mathrm{mOsm} / \mathrm{L}$ seems to have arisen from a 1972 study published by Becker and Vries ${ }^{5}$ in which they report a case series of 18 patients, half of whom required serum osmolalities $>320 \mathrm{mOsm} / \mathrm{L}$ to control their intracranial pressure. Eight of the 9 patients whose osmolalities exceeded $320 \mathrm{mOsm} / \mathrm{L}$ died. Three of these patients had renal failure and 5 had "systemic acidosis." The sodium limit of $155 \mathrm{mEq} / \mathrm{L}$ seems to have been derived from the work of Bingham, ${ }^{6}$ which was published in 1986. In this paper, Bingham reported a series of 18 patients and noted a high mortality rate in those patients whose serum sodium values exceeded the $155 \mathrm{mEq} / \mathrm{L}$ threshold. Neither of these studies provides evidence that would be considered convincing by current standards, despite their lasting influence. Of note, the risk of renal failure has been of prime importance in establishing these thresholds.

Evidence suggesting that more liberal thresholds are safe for hypertonic saline seems to have arisen from two publications from the same institution. ${ }^{12,17}$ These papers influenced the BTF's first pediatric guidelines, ${ }^{1}$ and this seems to be how more liberal administration limits for hypertonic saline became popularized. Notably, though, these more liberal thresholds were immediately questioned $^{9}$ and are not universally used. Clearly, administration limits could benefit from additional investigation.

So what should we do while we're waiting for more research? In considering the new data from Vedantam et al. in the context of the broader literature, I think we need to be more thoughtful about raising serum sodium values above $142 \mathrm{mmol} / \mathrm{L}$, and we should consider being less liberal with hyperosmolar therapies. New investigations aiming to optimize our administration of hyperosmolar therapies are clearly needed, however. The Transforming Research and Clinical Knowledge (TRACK) and Collaborative European Neurotrauma Effectiveness Research (CENTER) TBI studies will hopefully be very informative in this regard. Although clinicians increasingly dismiss animal investigations, we should consider that the ability to perform controlled experimentation and to examine histological data from animal models may bring forth key data informing the use of hyperosmolar therapy in clinical care, which is very difficult to derive from human study.

https://thejns.org/doi/abs/10.3171/2017.8.FOCUS17506

\section{References}

1. Adelson PD, Bratton SL, Carney NA, Chesnut RM, du Coudray HE, Goldstein B, et al: Guidelines for the acute medical management of severe traumatic brain injury in infants, children, and adolescents. Chapter 11. Use of hyperosmolar therapy in the management of severe pediatric traumatic brain injury. Pediatr Crit Care Med 4 (3 Suppl):S40-S44, 2003

2. Aiyagari V, Deibert E, Diringer MN: Hypernatremia in the neurologic intensive care unit: how high is too high? J Crit Care 21:163-172, 2006

3. American College of Surgeons Committee on Trauma: ACS
TQIP Best Practices in the Management of Traumatic Brain Injury. Chicago: American College of Surgeons, 2015 (https://www.facs.org/ /media/files/quality\%20programs/ trauma/tqip/traumatic\%20brain\%20injury\%20guidelines. ashx) [Accessed September 7, 2017]

4. Badjatia N, Carney N, Crocco TJ, Fallat ME, Hennes HMA, Jagoda AS, et al: Guidelines for prehospital management of traumatic brain injury 2nd edition. Prehosp Emerg Care 12 (Suppl 1):S1-S52, 2008

5. Becker D, Vries V: The alleviation of increased intracranial pressure by the chronic administration of osmotic agents, in Brock M, Dietz H (eds): Intracranial Pressure. Berlin: Springer, 1972, pp 309-315

6. Bingham WF: The limits of cerebral dehydration in the treatment of head injury. Surg Neurol 25:340-345, 1986

7. Brain Trauma Foundation, American Association of Neurological Surgeons, Joint Section on Neurotrauma and Critical Care: Use of mannitol. J Neurotrauma 17:521-525, 2000

8. Bratton SL, Chestnut RM, Ghajar J, McConnell Hammond FF, Harris OA, Hartl R, et al: Guidelines for the management of severe traumatic brain injury. II. Hyperosmolar therapy. J Neurotrauma 24 (Suppl 1):S14-S20, 2007 (Erratum in J Neurotrauma 25:276-278, 2008)

9. Dominguez TE, Priestley MA, Huh JW: Caution should be exercised when maintaining a serum sodium level $>160$ meq/L. Crit Care Med 32:1438-1440, 2004

10. Gullans SR, Verbalis JG: Control of brain volume during hyperosmolar and hypoosmolar conditions. Annu Rev Med 44:289-301, 1993

11. Kellie G: Appearances observed in the dissection of two individuals; death from cold and congestion of the brain. Trans Med Chir Sci Edinb 1:84-169, 1824

12. Khanna S, Davis D, Peterson B, Fisher B, Tung H, O'Quigley $\mathrm{J}$, et al: Use of hypertonic saline in the treatment of severe refractory posttraumatic intracranial hypertension in pediatric traumatic brain injury. Crit Care Med 28:1144-1151, 2000

13. Li M, Hu YH, Chen G: Hypernatremia severity and the risk of death after traumatic brain injury. Injury 44:1213-1218, 2013

14. Lien YH, Shapiro JI, Chan L: Effects of hypernatremia on organic brain osmoles. J Clin Invest 85:1427-1435, 1990

15. Maggiore U, Picetti E, Antonucci E, Parenti E, Regolisti G, Mergoni M, et al: The relation between the incidence of hypernatremia and mortality in patients with severe traumatic brain injury. Crit Care 13:R110, 2009

16. Monro A: Observations on the Structure and Function of the Nervous System. Edinburgh: Creech \& Johnson, 1783

17. Peterson B, Khanna S, Fisher B, Marshall L: Prolonged hypernatremia controls elevated intracranial pressure in headinjured pediatric patients. Crit Care Med 28:1136-1143, 2000

18. Van Beek JGM, Mushkudiani NA, Steyerberg EW, Butcher I, McHugh GS, Lu J, et al: Prognostic value of admission laboratory parameters in traumatic brain injury: results from the IMPACT study. J Neurotrauma 24:315-328, 2007

19. Vedantam A, Robertson CS, Gopinath SP: Morbidity and mortality associated with hypernatremia in patients with severe traumatic brain injury. Neurosurg Focus 43(5):E2, 2017

20. Wilkins RH, Weed LH, McKibben PS: Neurosurgical classic-XXXII. Experimental alteration of brain bulk. J Neurosurg 22:404-419, 1965

\section{Disclosures}

The author reports no conflict of interest. 